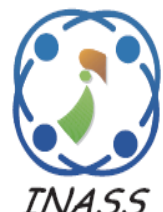

\title{
Osteoporosis Detection in Lumbar Spine L1-L4 Based on Trabecular Bone Texture Features
}

\author{
Kavita Avinash Patil ${ }^{1 *}$ \\ K. V. Mahendra Prashanth ${ }^{2}$ \\ A Ramalingaiah ${ }^{3}$ \\ ${ }^{I}$ Department of Electronics \& Communication Engineering, Sri Jagadguru Balagangadhranatha Institute of \\ Technology, Faculty, New Horizon College of Engineering, \\ Affiliated to Visvesvaraya Technological University, Bangalore, India. \\ ${ }^{2}$ Department of Electronics and Communication Engineering, Sri Jagadguru Balagangadhranatha Institute of \\ Technology, Affiliated to Visvesvaraya Technological University, Bangalore, India. \\ ${ }^{3}$ A Ramalingaiah M.S (ortho) Orthopedic Doctor, India. \\ * Corresponding Author’s Email: kavitamalagatti@gmail.com
}

\begin{abstract}
The proposed work can be divided into two parts: first, remove the noise from the trabecular lumbar spine L1-L4 of the X-ray images using two-stage principal component analysis with neighbourhood pixel grouping, followed by a hybrid median filter, and the texture features are enhanced with sharpening combined with range filters. Second, detect osteoporosis using texture features. This can be done by one-dimensional discrete wavelet transform, followed by a two-dimensional edge detection filter. Finally, classify the normal or osteoporotic images according to conventional classifications. Testing is conducted using X-ray images and dual-energy X-ray absorptiometry (DXA) reports from the same person. The DXA report describes a statistical analysis of normal or osteoporotic, but the proposed work is classified as osteoporotic or normal according to the texture features. Classified results are validated with the DXA and provide an average accuracy of $99.18 \%$. The proposed method has better diagnostic accuracy than the existing method using DXA with X-ray.
\end{abstract}

Keywords: De-noising, Lumbar spine, NPG-PCA, Texture features, Trabecular bone, X-ray images.

\section{Introduction}

Detection of Osteoporosis in the Lumbar spine from L1-L4 using X-ray images poses a challenge because it is a silent bone disease where bone become exceptionally frail and bound to break [1]. It regularly creates unnoticed over the years, without any indications or inconvenience until a bone break. Cracks brought about by osteoporosis regularly happen in the lumbar spine from L1-L4. Certainly, pre-processing is required because the noise will be present in the X-ray images during the acquisition process and de-noising with enhancement techniques are the basic step to get better image quality for further process. Numerous de-noising schemes are widely considered as smoothening filters both in spatial as well as frequency domain strategies [2]. Later part de-noising schemes are developed based on wavelet [3], curvelet [4], and ridgelets [5]. With the fast advancement of current computerized image processing and its wide applications in today's life, pre-processing is necessary for a higher quality of the image. The osteoporosis detection of thoraco-lumbar spine using computed tomography (CT) images [6] by considering the texture features based on fractal analysis to obtain feature descriptors. The normal and abnormal bone classification depends on the feature descriptors using different classifiers. The fractal analysis alone not able to achieve better accuracy, but the combination of fractal analysis with other methods can improve the system accuracy. Detection of Osteoporosis [7] of lumbar spine CT in terms of HU (Hounsfield units) with additional QCT information using machine learning (ML) tools. Numerous regression models with QCT information are used to detect non-osteoporotic or osteoporotic 
vertebra by computing the T-score inputs. The accuracy of the system is $92 \%$, however, there should be an improvement by analyzing larger sets of data. Efficient algorithms are needed to take the necessary part of the vertebral image in order to estimate the $\mathrm{BMD}$. The techniques are developed to assess bone density and $3 \mathrm{D}$ subject model of the lumbar spine using a single anteroposterior (AP) DEXA image [8]. A 3D density and statistical model was built utilizing a training set of QCT scans which was further between QCT and DXA derived estimation extended from 0.81-0.97. The model improved the detection of osteoporosis and fracture risk assessment better than the AP DXA scan without any further assessment. The method could hardly limited to local deformities, which in turn impact on the system accuracy. The recurrent neural network [9] was used to predict osteoporosis of the AP lumbar spine using Magnetic Resonance Imaging (MRI). There were some practical challenges for deep learning, for instance, a lower training process results in less system precision. Therefore, improving the system performance requires larger sets of data. Morphometric [10] features such as crush, wedge, and biconcave of lumbar spine CT images are extracted based on vertebral edges and contours to detect osteoporotic bone. The main advantage of the system is finding the vertebral compression fractures. The texture features [11] are extracted using a multifractal spectrum machine learning model to diagnose osteoporosis of spine MRI and CT images. The system making more complex for diagnosing. The features such as volumetric parameter (morphological) and bone density determinants [12] are used to classify Vertebral Compression Fractures (VCF) of Osteoporotic origin of CT images, which provides better classification accuracy. The model gives better shape analysis to discriminate between fractured and normal vertebral [13] using MRI. Various texture and shape features with semiautomated segmentation of lumbar vertebrae must enhance the complete processing chain leading to better classification in the diagnosis of VCFs benign versus malignant. The hybrid type classifier model [14] is developed to diagnose osteoporosis using an artificial neural network (ANN) based monarch butterfly optimization (MBO) technique built on individual attribute values like trabecular separation, trabecular number, age, body mass index, etc. The outcomes of the model are better analogized using receiver operating characteristics (ROC). However, it is applicable for especially high dimensional sets of data. Developed a system to classify osteoporosis and osteopenia of lumbar spine X-ray images using a deep convolutional neural network (DCNN) algorithm [15]. Three classes of DCNN were trained to classify, osteoporosis, osteopenia, and normal BMD. The deep learning model calculations are very challenging to bring accuracy into the system. DCNN was trained with PTLR (Plain thoraco lumbar radiography) images to identify vertebral fractures (VFs) [16]. The DCNN model helps in clinical trials for the early identification of VFs and prevents further invasive involvements. The model is not able to identify the fracture levels. The combination of three-dimensional texture features extracted using wavelets, local binary patterns, histogram of gradients, gray-level co-occurrence matrix features, and local volumetric BMD of CT images to detect osteoporosis [17]. To predict fractures appropriately more effective algorithms are required. The motive is to obtain accurately Dexa scores of the Lumbar region using CT based on ML algorithms [18]. The regression-based model is developed to resolve the correlation between DEXA T-score and grade calculation for L1-L4. It is a more complicated system for the analysis of osteoporosis. Automatic detection of osteoporosis in the lumbar vertebra using CT image based on the convolutional neural network (CNN) [19] consisting of two methodologies namely 1) mark segmentation 2) bone conditions classification network. The system needs additional image features as well as some of the clinical characters to verify the conditions of bone. Explored different machine learning models to forecast BMD utilizing DEXA and X-ray image features of the spine obtained by three Deep learning algorithms [20]. To improve the system performance, other ML algorithms with larger datasets are required. A quantitative study on the detection of lumbar spine osteoporosis using MRI-based score (M-score) [21] and correlation between lumbar spine signal intensity measured from MRI. The statistical quantitative method significantly improves to distinguish osteoporotic from non-osteoporotic bones. The threshold setting for $\mathrm{M}$-score is challenging, since it is varying for every sample with different SNRs.

In order to assess osteoporosis in the lumbar spine L1-L4, this paper presented an efficient model by dividing it into two parts. First is the preprocessing part of the model, needed to ensure the quality of X-ray images, and the second part is detecting osteoporosis in the lumbar spine L1-L4 using X-ray images as shown in Fig. 1. In the preprocessing step, the noise present in the $\mathrm{X}$-ray images is eliminated and the trabecular bone texture features are enhanced in order to visualize fine texture in the images. An approach to noise reduction based on two phases of neighbourhood pixel grouping (NPG)-PCA [27] followed by a hybrid median filter (HMF). To 


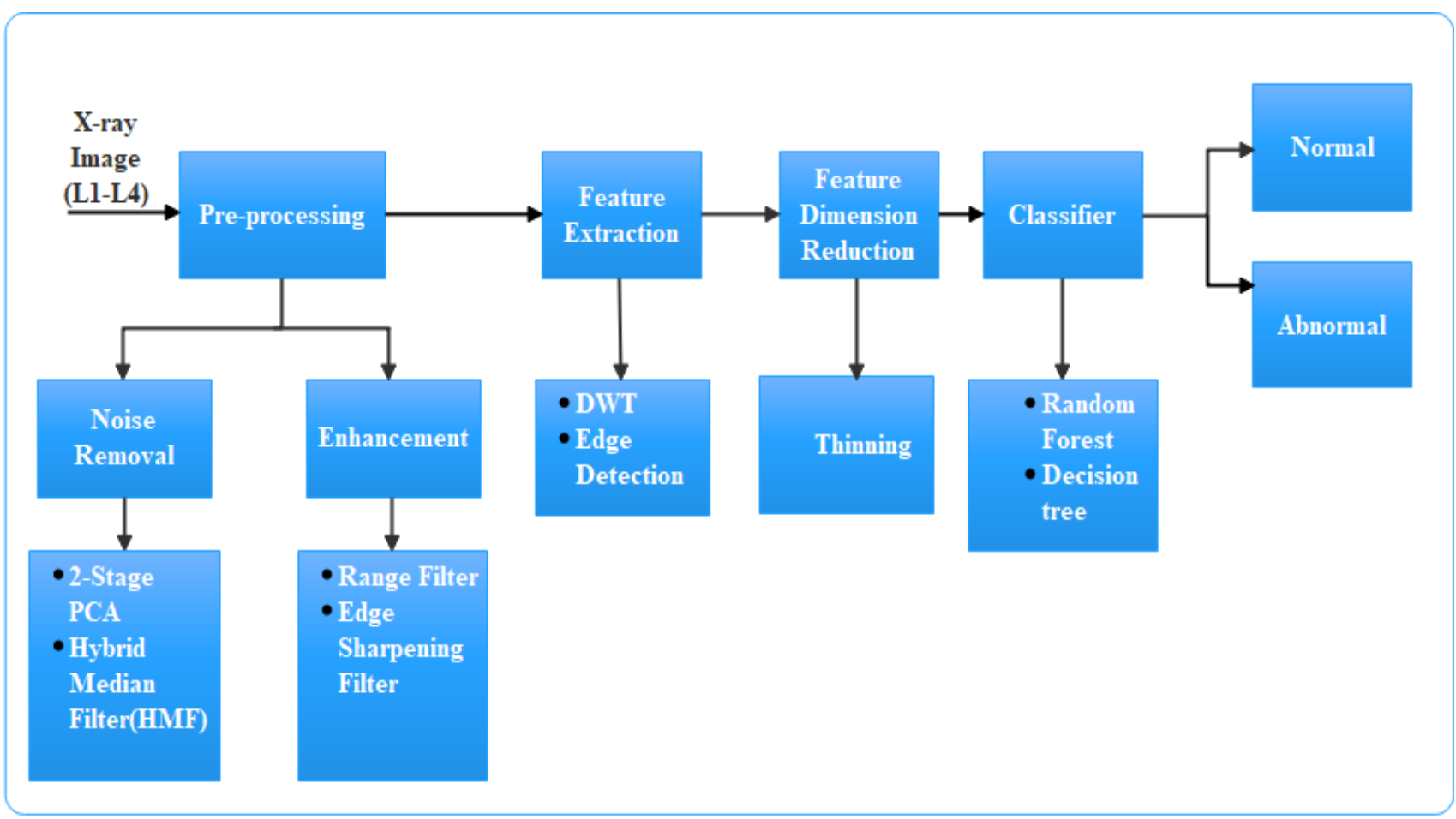

Figure. 1 Proposed system model

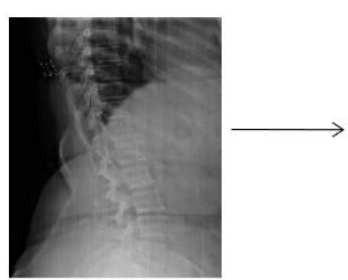

Normal LS View

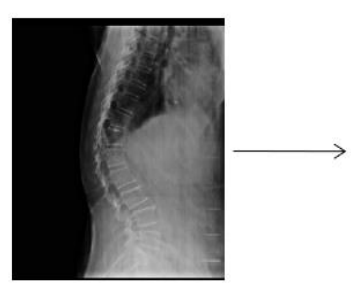

Abnormal LS View
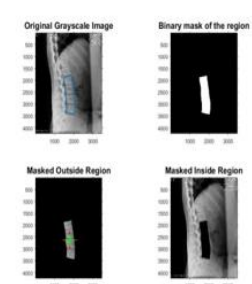

Free hand drawing tool
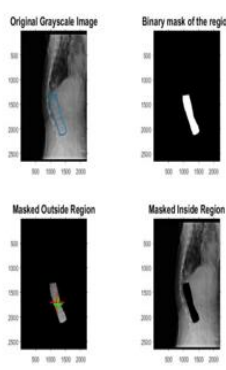

Free hand drawing tool
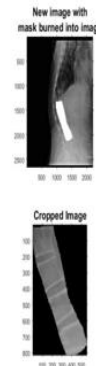

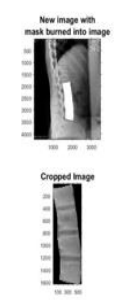

Figure. 2 Data preparation process

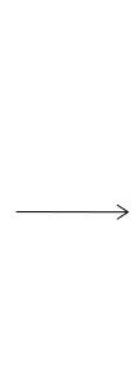

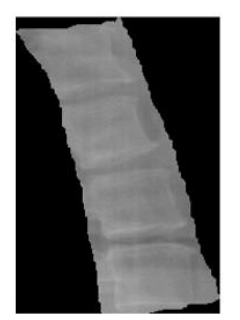

Lumbar Vertebral Image L1-L4

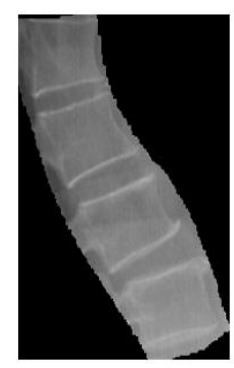

Lumbar Vertebral Image L1-L4

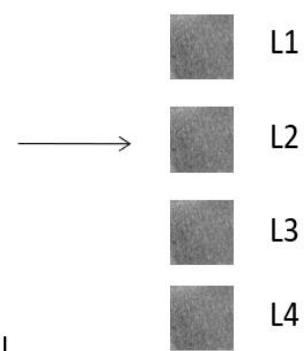

Trabecular Microarchitecture of Lumbar Vertebrae L1-L4 X-ray

image
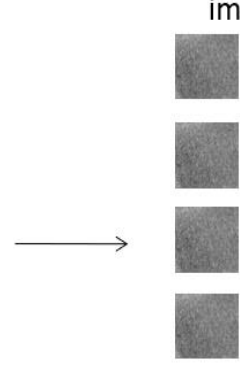

L1

L2

L3

Ł4

Trabecular

Microarchitecture of Lumbar Vertebral L1-L4 X-

ray image further enhance the texture, apply a sharpening filter followed by a range filter. A second part of the system identifies osteoporosis based on texture features. In this work, the features are extracted using a two-dimensional discrete wavelet transforms (2D-
DWT). In addition to highlighting the critical edges, 2D-edge detection filters are used so as to reduce the size of the fine features. Reduced texture features histograms are used as the supervised database to classify the input test X-ray images as normal or 
abnormal (osteoporosis) using conventional classification techniques.

Here are the main contributions of each section 2 describes how to pre-process lumbar spine X-ray images in order to improve image quality. Section 3 proposes a novel model in which osteoporosis is detected using image texture analysis. A detailed simulation study and discussion is provided in section 4 using the authenticated dataset. The paper's results and future work insights are summarized in section 5 .

\subsection{Data preparation}

Fig. 2 illustrates the data preparation process; the algorithm is as follows:

- Read the input X-ray lateral spine view image.

- Create a binary image (mask) from the region of interested section. Display the freehand mask.

- Label the binary image and compute the centroid and center of mass. Calculate the area, in pixels, that they drew.

- Get the $\mathrm{x}$ and $\mathrm{y}$ coordinates of the boundary of the freehand drawn region, get $n$ by $2 \mathrm{D}$ array of matrix.

- Plot over original image, don't drive away the image, force it to draw immediately.

- Disconnect the line into image by setting it to 255 wherever the mask is true.

- Display the image with the mask with disconnected region, mask the image and display it.

- keep only the part of the image that's inside the mask, zero outside mask.

- Now do the same but blacken inside the region.

- Now crop the image, display cropped image and write cropped Image.

\section{System model of pre-processing}

Pre-processing technique is a combination of denoising and enhancement of lumbar spine X-ray images from L1-L4. Two-phase PCA is utilized to eliminate the maximum amount of noise and also smoothen the edges in the images. And in the subsequent stage, the HMF is utilized to eliminate the noise at the edges. HMF uses a linear window to protect the subtleties edges and lines in the images. The edge sharpening filter followed with range filter are utilized to enhance the texture features in the edges, which are smoothened by the PCA.

\subsection{Block based de-noising}

De-noising based on the NPG-PCA consists of input $\mathrm{X}$-ray image dataset $\mathbf{X}_{\mathrm{v}}$, every element $\mathrm{x}_{\mathrm{k}}^{\mathrm{v}}$, where $\mathrm{k}=1,2 \ldots \mathrm{m}$, of the variable $\mathrm{X}_{\mathrm{V}}$ has $n$ number of samples. And it is represented by $\mathrm{X}_{\mathrm{k}}^{\mathrm{v}}$, the row vector consists of $n$ number samples of $\mathrm{x}_{\mathrm{k}}^{\mathrm{V}}$. Subsequently the dataset $X_{v}$ can be represented as $X_{v}=$ $\left(\left(X_{1}^{\mathrm{v}}\right)^{\mathrm{T}},\left(\mathrm{X}_{2}^{\mathrm{v}}\right)^{\mathrm{T}} \ldots\left(\mathrm{X}_{\mathrm{m}}^{\mathrm{v}}\right)^{\mathrm{T}}\right)^{\mathrm{T}}$.

Then the corresponding noisy image is described in Eq. (1).

$$
\mathbf{X}_{\mathrm{v}}=\mathrm{X}+\mathrm{V}
$$

and $\mathrm{X}=\left(\left(\mathrm{X}_{1}\right)^{\mathrm{T}},\left(\mathrm{X}_{2}\right)^{\mathrm{T}} \ldots\left(\mathrm{X}_{\mathrm{m}}\right)^{\mathrm{T}}\right)^{\mathrm{T}}, \mathrm{X}_{\mathrm{k}}$ is the row vector containing the $n$ number of samples with $\mathrm{V}=\left(\left(\mathrm{V}_{1}\right)^{\mathrm{T}},\left(\mathrm{V}_{2}\right)^{\mathrm{T}} \ldots\left(\mathrm{V}_{\mathrm{m}}\right)^{\mathrm{T}}\right)^{\mathrm{T}}$ is the noise row vector variable $\mathrm{V}_{\mathrm{k}}$ of $n$ number of samples. After that the centralized datasets of $\mathbf{X}_{\mathrm{V}}$ and $\mathrm{X}$ are obtained as in Eq. (2).

$$
\overline{\mathrm{X}}_{\mathrm{v}}=\overline{\mathrm{X}}+\mathrm{V}
$$

Where $\overline{\mathbf{X}}_{\mathrm{v}}, \overline{\mathrm{X}}$ are represented with as zero-mean i.e. subtracted from their respective mean values but noise vectors are considered as zero mean.

Computing the covariance matrix $\mathbf{K}_{\overline{\mathrm{X}}}$ of the $\overline{\mathrm{X}}$ and noise vector $\mathbf{K}_{\mathrm{V}}$. The covariance matrix of the noisy image is as in Eq. (3).

$$
\mathbf{K}_{\overline{\mathrm{X}}_{\mathrm{V}}}=\mathbf{K}_{\overline{\mathrm{X}}}+\mathbf{K}_{\mathrm{V}}
$$

Where $\mathbf{K}_{\overline{\mathrm{X}}}=\frac{\overline{\mathrm{X}} \overline{\mathrm{X}}^{\mathrm{T}}}{\mathrm{n}}$ and knowing that $\mathbf{K}_{\mathrm{V}}$ is a diagonal matrix $m \times m$ with its diagonal components is $\sigma^{2}$.

Subsequently, PCA transformation matrix $\mathbf{P}_{\overline{\mathrm{X}}}$ of the $\overline{\mathrm{X}}$ is related with $\mathbf{K}_{\overline{\mathrm{X}}}$ is the identical as the PCA transform matrix related with $\mathbf{K}_{\bar{X}_{\mathrm{V}}}$. Then the PCA decompose of $\mathbf{K}_{\overline{\mathrm{X}}}$ as in Eq. (4).

$$
\mathbf{K}_{\overline{\mathrm{X}}}=\mathbf{E}_{\overline{\mathrm{X}}} \mathbf{D}_{\overline{\mathrm{X}}} \mathbf{E}_{\overline{\mathrm{X}}}^{\mathrm{T}}
$$

where $\mathbf{E}_{\overline{\mathrm{X}}}$ is the ortho-normal Eigen vector-matrix of $m \times m, \mathbf{D}_{\overline{\mathrm{X}}}$ is the $m \times m$ eigen value diagonal matrix and $\mathbf{E}_{\overline{\mathrm{X}}}^{\mathrm{T}}$ is the transpose matrix. Then $\mathbf{K}_{\mathrm{V}}$ can be written as in Eq. (5).

$$
\mathbf{K}_{\mathrm{V}}=\mathbf{E}_{\overline{\mathrm{X}}} \mathbf{I} \sigma^{2} \mathbf{E}_{\overline{\mathrm{X}}}^{\mathrm{T}}
$$

Then the covariance matrix of $\mathbf{X}_{\mathrm{v}}$ can be written it as in Eq. (6). 


$$
\begin{aligned}
& \mathbf{K}_{\overline{\mathrm{X}}_{\mathrm{V}}}=\mathbf{E}_{\overline{\mathrm{X}}} \mathbf{D}_{\overline{\mathrm{X}}} \mathbf{E}_{\overline{\mathrm{X}}}^{\mathrm{T}}+\mathbf{E}_{\overline{\mathrm{X}}} \mathbf{I} \sigma^{2} \mathbf{E}_{\overline{\mathrm{X}}}^{\mathrm{T}} \\
& \mathbf{K}_{\overline{\mathrm{X}}_{\mathrm{V}}}=\mathbf{E}_{\overline{\mathrm{X}}}\left(\mathbf{D}_{\overline{\mathrm{X}}}+\mathbf{I} \sigma^{2}\right) \mathbf{E}_{\overline{\mathrm{X}}}^{\mathrm{T}} \\
& \mathbf{K}_{\overline{\mathrm{X}}_{\mathrm{V}}}=\mathbf{E}_{\overline{\mathrm{X}}}\left(\mathbf{D}_{\overline{\mathrm{X}}_{\mathrm{V}}}\right) \mathbf{E}_{\overline{\mathrm{X}}}^{\mathrm{T}}
\end{aligned}
$$

The Eq. (7). implies that both $\mathbf{K}_{\bar{X}_{V}}$ and comprise the identical Eigen vector matrix $\mathbf{E}_{\overline{\mathrm{X}}}$.Therefore, practical implementation can straightly compute $\mathbf{E}_{\overline{\mathrm{X}}}$ by decomposing $\mathbf{K}_{\bar{X}_{\mathrm{V}}}$, and after that the orthonormal PCA transform matrix for $\bar{X}$ can be calculated in Eq. (8). is

$$
\mathbf{P}_{\overline{\mathrm{X}}}=\mathbf{E}_{\overline{\mathrm{X}}}^{\mathrm{T}}
$$

Apply $\mathbf{P}_{\bar{X}}$ to $\overline{\mathbf{X}}_{\mathrm{V}}$ dataset, thus transformed PCA domain $\overline{\mathbf{Y}}_{\mathrm{v}}$ vector matrix as in Eq. (9).

$$
\overline{\mathbf{Y}}_{\mathrm{V}}=\mathbf{P}_{\overline{\mathrm{X}}} \overline{\mathbf{X}}_{\mathrm{V}}=\mathbf{P}_{\overline{\mathrm{X}}} \overline{\mathbf{X}}+\mathbf{P}_{\overline{\mathrm{X}}} \overline{\mathbf{V}}=\overline{\mathbf{Y}}+\mathbf{V}_{\mathrm{Y}}
$$

The covariance matrix of $\overline{\mathbf{Y}}_{\mathrm{V}}$ is

$$
\mathbf{K}_{\overline{\mathrm{Y}}_{\mathrm{V}}}=\frac{\overline{\mathbf{Y}}_{\mathrm{V}} \overline{\mathbf{Y}}_{\mathrm{V}}^{\mathrm{T}}}{\mathrm{n}}=\mathbf{K}_{\overline{\mathrm{Y}}}+\mathbf{K}_{\mathrm{V}_{\mathrm{Y}}} \text {. }
$$

The $\overline{\mathbf{Y}}_{\mathrm{V}}$ contains most of the important energy with noiseless data sets are concentrated on the several components, whereas the energy of noise dispenses much more consistently. The noise present in the $\overline{\mathbf{Y}}_{\mathrm{v} \text { can be able to be suppressed by means of linear }}$ minimum mean square error estimation (LMMSE) method. In view of the fact that $\overline{\mathbf{Y}}_{\mathrm{v}}$ is centralized, the LMMSE of $\overline{\mathbf{Y}}_{\mathrm{i}}$ is the $\mathrm{i}^{\text {th }}$ row of the dataset which is obtained using Eq. (10).

$$
\overline{\mathbf{Y}}_{\mathrm{i}}=\mathrm{W}_{\mathrm{i}} \overline{\mathrm{Y}}_{\mathrm{v}}^{\mathrm{i}}
$$

The $\mathrm{W}_{\mathrm{i}}$ is defined as shrinkage component and it is obtained from the covariance matrix

$\mathrm{W}_{\mathrm{i}}=\frac{\mathbf{K}_{\overline{\mathrm{Y}}}(\mathrm{i}, \mathrm{i})}{\left(\mathbf{K}_{\overline{\mathrm{Y}}}(\mathrm{i}, \mathrm{i})+\mathbf{K}_{\mathrm{V}_{\mathrm{Y}}}(\mathrm{i}, \mathrm{i})\right)}$ and $\overline{\mathrm{Y}}_{\mathrm{v}}^{\mathrm{i}}$ is the $\mathrm{i}^{\text {th }}$ row of $\overline{\mathbf{Y}}_{\mathrm{v} . \mathrm{In}}$ the flat regions, $\mathbf{K}_{\bar{Y}}(i, i)$ is a large extent to smaller than $\mathbf{K}_{\mathrm{V}_{\mathrm{Y}}}$, in order that $\mathrm{W}_{\mathrm{i}}$ is closer to zero. Consequently, most part of the noise will be suppressed. $\widehat{\overline{\mathrm{Y}}}$ matrix is represented as the entire $\overline{\mathbf{Y}}_{\mathrm{i}}$. By transforming back to the time domain to get a denoised dataset from Eq. (11).

$$
\widehat{\overline{\mathbf{X}}}=\mathbf{P}_{\overline{\mathbf{X}}}^{\mathrm{T}} \widehat{\widehat{\mathbf{Y}}}
$$

The de-noised $\widehat{\mathbf{X}}$ dataset is obtained by adding mean values to $\widehat{\overline{\mathbf{X}}}$, and hence the above-mentioned procedure is to apply for each pixel to get the complete de-noised image. There are two primary reasons for the residual noise present in the first phase of PCA. Foremost, the original dataset $\mathbf{X}_{\mathrm{V}}$ contains the strong noise and the covariance matrix $\mathbf{K}_{\bar{X}_{V}}$ is corrupted with a large amount of noise, which results to assess the preconception of the transformation matrix. For this reason, the performance of the denoising process deteriorates. Subsequently, the strong noise will also introduce errors into NPG. Hence, it is essential to process in the second phase of PCA, to get a better reduction in noise level with a similar procedure. The majority of the noise will be eliminated by utilizing the de-noising techniques in the first phase. However, there is still a lot of pleasant residual noise in the output of the first phase. The denoised image by utilizing the proposed NPG-PCA strategy although the peak to signal to noise ratio (PSNR) is much improved, but still residual noise observed. There are primarily two purposes behind the residual noise. First, the original dataset $\mathbf{X}_{\mathrm{V}}$ itself have strong noise, and its $\mathbf{K}_{\mathrm{V}}$ covariance matrix is also corrupted, which prompts in the assessment of the PCA and subsequently weakens the performance of the first phase. Second, it also introduces noise in the NPG process by which, estimation of the covariance matrix affects. Therefore, the second phase of PCA is necessary to eliminate the noise. The two-phase PCA output contains a certain quantity of noise at the edges. This noise is diminishing by means of HMF, which is typically non-linear in nature. Especially it decreases the impulse type of noise by safeguarding the edges and lines in the images. It operates in three steps: pixels from different directions are categorized separately, three different median values are estimated: the horizontal and vertical pixels median $\mathrm{M}$ and diagonal pixels $\mathrm{M}_{\mathrm{d}}$, the filtered value at the central pixel $\mathrm{C}$ of $5 \times 5 \mathrm{sub}$ image is the median $\left(M, M_{d}, C\right)$, the entire filter output is achieved by shifting $5 \times 5$ window in the entire image. This filter does not smoothen the details in the image but usually offers better image quality. One basic advantage of this filter is its adaptive nature, so as to allows better removal noise in small spatial window.

\subsection{Enhancement}

The edge sharpening and range filters are utilized to enhance the texture features of the X-ray image, which is smoothened by the de-noise stages. The edge sharpening filter kernel window size $3 \times 3$ is as shown in Fig.3. The entire output filter is attained 


\begin{tabular}{|c|c|c|}
\hline 0 & -1 & 0 \\
\hline-1 & 5 & -1 \\
\hline 0 & -1 & 0 \\
\hline
\end{tabular}

Figure.3 Filter kernel

by shifting kernel along with the image. In the final stage of pre-processing range filter is employed to maintain every pixel output reach within the specified value. For example, maximum value to least estimation values are assessed in the $3 \times 3$ local structure around the relating pixel in the input image. Only the vertical edges are characterizing in the range filters since the vertical variations are more significant in the enhancement of the image texture features. For this situation, the local returns an enhanced pixel, when there is an more contrast variations between pixel in the specified window in the input pixel, which are participated in the filtering process. The enhanced stage retains the fine texture features for further processing.

\subsection{Pre-processing agorithm}

- NPG - PCA is processing with assuming white Gaussian noise added into the input Xray images.

- Divide the image into overlapping blocks of the size $\mathrm{S} \times \mathrm{S}$ vector: variable block and $\mathrm{T} \times \mathrm{T}$ vector: training block.

- $\mathrm{S}<\mathrm{T}, \mathrm{S}$ is inside the training block $\mathrm{T}$, set as $\mathrm{S}=3$ and $\mathrm{T}=9$.

- Essentially the de-noised pixel is the centered pixel in the $S$.

- The $\mathrm{T} \times \mathrm{T}$ vector is taken as: $\mathrm{T}_{2}$ long vector, collected as possible $\mathrm{S}$ vectors in the $\mathrm{T}$.

- Compute the PCA de-composition using Eq. (4) for S vectors.

- Consider the projections of the training vectors on to the PCA.

- Find the variance of the PCA coefficients as $\sigma^{2}$.

- Reconstruct the de-noised using Eq. (4). in training vectors $\mathrm{T}$. Since vectors $\mathrm{S}$ in $\mathrm{T}$ are overlapped, taking averages in overlap regions after the de-noised training vectors are placed back in the train region.

- Each one of pixel in the training block is estimated $\mathrm{T}_{2}$ times, consequently, the restored pixel value will be the average of the entire estimated value, obtained from all windows including the pixels. This process of estimation carried out by shifting the window in the entire image.

- After two phase NPG - PCA followed with application of HMF as per in the section 2.1 by considering $5 \times 5$ sub-image and execute this by shifting the window in the entire image.

- Enhancement of the image is carried out as per in the section 2.2 to improve the texture features in the X-ray image using Fig. 3. filter kernel with range filter.

\section{System model of osteoporosis detection}

The proposed system model of osteoporosis detection is split into two parts: texture feature extraction and classification. The suggested system model reduces the dimension of the feature vector so as to achieve osteoporosis detection effectively. The reduction in feature dimension by considering only the horizontal coefficients $\mathrm{H}_{\mathrm{C}}$ along with vertical coefficients $\mathrm{V}_{\mathrm{C}}$ in the 2D-DWT. Further just edge Information and pixel adjustment are done to maintain a strategic distance from vulnerability in the pixel. In this work, different wavelet families are used to extract the texture features in order to get significant information. The wavelet transformation-based feature extraction is a unique type, which causes orientation in the texture information in various directions. Further, the 2-D edge detecting filter is employed to further extract the fine texture information with reduction in the feature dimension. Only the texture feature histogram bins are considered in the supervised database, which in turn reduces the feature dimension. The different classifiers are used to classify the given test X-ray image as normal or abnormal.

\subsection{DWT}

The DWT has accomplished broad endorsed in the analysis of images, which characterizes a set of small basis wave functions. These wave functions are considered as wavelets, so as to acquire one wavelet called fundamental wave by translation and scaling. It has been started as an efficient and versatile strategy for independent band divisions in the image. The coefficients of wavelet are all together adequately useful towards the investigation of the texture features in the image, which provide sufficient information. Huge texture feature data is gathered within particular wavelet coefficients, which are keeping in a particular band of data in the pixels. The proposed 2D-DWT [22, 2] technique utilizes single-level decomposition for the input image matrix, which permits investigating texture 
data at various scales. The decomposition restores the average coefficient matrix and complete detailed coefficient matrix. For example, complete coefficient matrices are vertical, horizontal, and diagonal. The only horizontal coefficients and vertical coefficients matrices are utilized in the processes of featureextraction. These two are playing an important role in the analysis of the edges, ridges, and blobs present in the texture image. And subsequently, at this stage, the proposed technique decreases the half of the texture features component in the given image.

\subsection{Edge detection filter}

Further to identify the edges in $\mathrm{H}_{\mathrm{C}}$ and $\mathrm{V}_{\mathrm{C}}$, the proposed two-dimensional edge detection filter algorithm is applied to hold the significant texture features, which further decreases the features dimension dramatically. While conventional Canny edge locality gives generally basic yet exact technique for edge identification issue, with additionally requesting necessities on the precision and strength on the detection. The customary algorithm can presently don't deal with the difficult edge recognition task. The principle imperfections of the conventional algorithm [23, 2] leads to the following problems: It will construct the chance of missed frail edges, the presence of disconnected boundaries in the outcome, responsive to the noise in turn can simply identify the artificial edges, moreover, misplace genuine edges due to gradient amplitude calculation, which is computed from the finite difference mean value in the small locality window and two unchanging global threshold values result in false edges. However, it makes complex, but different neighborhood regions will require different threshold assessments to detect real edges more precisely [31] . Especially, the proposed 2-D edge detection filter algorithm uses multi-step to extract texture features. The Gaussian function and it's firstorder derivatives are used to highlights the edge regions within the filtering kernel. The kernel is defined in Eq. (12). The following steps are carried out to bring the fine texture information in the image.

$$
\begin{aligned}
& h_{X}(x, y)=g(x, y) \times g^{\prime}(x, y) \\
& g(x, y)=\frac{1}{\sqrt{2 \pi \sigma^{2}}} \exp \left(\frac{x^{2}-y^{2}}{\sigma^{2}}\right) \\
& g^{\prime}(x, y)=-\frac{x}{\sqrt{2 \pi \sigma^{3}}} \exp \left(-\frac{x^{2}-y^{2}}{\sigma^{2}}\right)
\end{aligned}
$$

STEP -1: The $h_{x}(x, y)$ is the impulse response of the filter, which is applied to $\mathrm{H}_{\mathrm{C}}$ in Eq. (13). to get the texture information variations along the horizontal direction in the image and $*$ indicates convolution.

$$
\mathrm{I}_{\mathrm{X}}=\mathrm{h}_{\mathrm{X}}(\mathrm{x}, \mathrm{y}) * \mathrm{H}_{\mathrm{C}}
$$

Similarly, the $\mathrm{h}_{\mathrm{y}}(\mathrm{x}, \mathrm{y})$ is the impulse response of the filter which is applied to $V_{C}$ in Eq. (14). to get the texture information variations along the vertical directions in the image.

$$
\mathrm{I}_{\mathrm{Y}}=\mathrm{h}_{\mathrm{Y}}\left(\mathrm{x}, \mathrm{y}, \theta_{\mathrm{Y}}\right) * \mathrm{~V}_{\mathrm{C}}
$$

Where $\mathrm{h}_{\mathrm{Y}}(\mathrm{x}, \mathrm{y})=\mathrm{g}(\mathrm{x}, \mathrm{y}) \times \mathrm{g}^{\prime}(\mathrm{x}, \mathrm{y})$

$g^{\prime}(x, y)=-\frac{y}{\sqrt{2 \pi \sigma^{3}}} \exp \left(-\frac{x^{2}-y^{2}}{\sigma^{2}}\right)$

The filter $h_{\mathrm{y}}(\mathrm{x}, \mathrm{y})$ is rotated by $\theta_{\mathrm{Y}}=\frac{\pi}{2}$.

STEP-2: Compute the gradient magnitude image of filtered responses as shown in Eq. (15).

$$
G=\sqrt{I_{X}^{2}+I_{Y}^{2}}
$$

and the direction of orientation of the texture information in the image is calculated in Eq. (16).

$$
\theta=\tan ^{-1} \frac{\mathrm{I}_{\mathrm{Y}}}{\mathrm{I}_{\mathrm{X}}}
$$

STEP-3: The $G$ and $\theta$ are able to analyze by means of a mixture of unlike edges, in addition to that $\theta$ and $\mathrm{G}$ manipulate the texture features. Further to determine the issues in some place that is inflexible on the way to empirically decide the two different threshold assessments. Otsu's method [31] can employ to create a high threshold value on the suppressed on-maximum $G$ image. But a low threshold value is characteristically must be to half of the highest threshold value for this situation. Since the $G$ image values are continuous in nature with no maximum values are defined within it. An Otsu's scheme has been used to estimate each data value and the occurrences of its entire histogram.

STEP-4: Although, conventional canny edge detection has a better detection outcome, but it is able to detect every edge not firmly. An arithmetical morphology is used in the direction of thinning identified edges in the image.

STEP-5: Finally, the trabecular bone texture feature histogram database is generated after thinning the image.

\subsection{Classifications}

Classification is one that grouping the belongings together on the foundation of some convinced regular features. It is actually the means of putting alike features into particular labeled groups. This paper introduced two different groups of images, 
one group is a normal image which is not affected from the osteoporosis diseases and another group is an abnormal image which is affected from the osteoporosis diseases. A particular abnormal situation so as to negatively affect the trabecular bone structure. Different classifications technique are available in machine learning, whereas classification refers to supervised learning which is a predictive modelling problem, where the class label is predicted for a given input data. K- Nearest Neighbour (Knn) is a straightforward calculation that performs regression or classification depends on the distances between them, for example, a classifier of affiliation. The multivariate technique used in Discriminant analysis (DA) [24] separates two or more groups of individuals depends on variables, calculated on each trial unit and discover the contribution of each variable in separating the groups. Naives Bayes [25] is one of the straightforward classifications, which usually shows lower variance and higher bias (A high bias means the prediction will be inaccurate). For uncomplicated datasets, it is a quick and easy classifier but not fit for the richer dataset. Decision Trees (DT) settle on their choices from root up to the branches, they are superior to Knn in disregarding insignificant features. However, they are inclined to over fitting, with which ensemble learning can assist. The Support Vector Machine (SVM) separates the information (data)space into two classes by means of hyper-planes. SVM's are acceptable at taking care of unpredictable, nonlinear situations, and tend not to over-fit. Random forest (RF) [26] give better performance in prediction Since it is modelled by using several DTs, it is termed as ensemble classifier. It generates number of classification trees and bootstrap model is utilized to train every tree in the training set data. It searches only for arbitrary subset variables to facilitate split at each and every node. To classify the input vector is given to every tree in RF, every tree chooses for a class. At last, RF select the class with majority of the votes. It has the capability to hold bigger input data-sets while contrast with other techniques.

\subsection{Osteoporosis detection algorithm}

- Evaluate the one-level decomposing of 2D DWT of the X-ray pre-processed image by utilizing the different wavelets.

- 2D-DWT gives the average coefficient matrix $A_{C}$ and complete detailed coefficient matrix like: horizontal $\mathrm{H}_{\mathrm{C}}$, vertical $\mathrm{V}_{\mathrm{C}}$ and diagonal $\mathrm{D}_{\mathrm{C}}$. Consider only $\mathrm{H}_{\mathrm{C}}$ and $\mathrm{V}_{\mathrm{C}}$.

- Compute the horizontal and vertical intensity variations of the image using Eq. (13). and
Eq. (14). respectively.

- Calculate the gradient and its orientated images using Eq. (15). and Eq. (16). respectively.

- Find two different threshold images are assessed empirically using Otsu's method using $\mathrm{G}$ and $\theta$.

- Estimate each data value depending on the threshold images and its occurrences of its entire histogram.

- Thinning the identified edges in the threshold images and finally obtained the texture feature image by taking the averages of the two thinning images.

- Take the histogram of the image data, which is used as supervised database to classify the input test X-ray image using classifiers, which are mentioned in the section 3.3 whether the given test image is a normal or abnormal.

\section{Experimental outcomes and discussions}

Images were obtained on Lumbar Vertebrae with a direct digital $\mathrm{X}$-ray prototype. The focal distance was set at $0.812 \mathrm{~m}$. The X-ray parameters were $75-80$ $\mathrm{kV}$ and $80 \mathrm{mAs}$ for all patients. The images used in this research have been provided by Dr. A Ramalingaiah (ortho) Orthopedic Doctor, Bangalore. No.271 Abhilasha Orthopaedic Hospital $5^{\text {th }}$ Block 100ft Rd Banashankari 3rd Stage Bangalore - 560085. The data consist of 108 2D radiographic images in JPEG format. Experimentally 52 control subjects(normal) and 56 pathological (Osteoporotic or abnormal) X-ray images along with DXA reports of the same person's certified datasets are collected to test the system. The DXA report describes each person's lumbar spine L1-L4 statistical analysis status of normal or osteoporotic.Table1 listed the region of interest (ROI) bone X-ray image database of the lumbar spine (L1-L4), which are considered to test the proposed system experimentally and along with the DXA report in this work.

\subsection{Pre-processing}

The implementation of two-phase NPG-PCA denoising actually centered the pixels within the block of the image to be de-noised. As a result, the denoised pixel value can be set as the average of all pixels in the window and same approach was used in [27]. Proposed techniques can reduce noise levels in a large part of input images with varying noise variances $\sigma^{2}$. HMF further reduces impulse noise at 
Table 1. Database description

\begin{tabular}{|c|c|c|c|c|}
\hline $\begin{array}{c}\text { X-ray } \\
\text { images } \\
\text { (L1-L4) }\end{array}$ & $\begin{array}{c}\text { No. of } \\
\text { training } \\
\text { samples }\end{array}$ & $\begin{array}{c}\text { No. of } \\
\text { testing } \\
\text { samples }\end{array}$ & $\begin{array}{l}\text { Total } \\
\text { no. of } \\
\text { sample } \\
\text { s }\end{array}$ & $\begin{array}{c}\text { No. of } \\
\text { DEXA } \\
\text { reports } \\
(\mathbf{1 0 8})\end{array}$ \\
\hline $\begin{array}{l}\text { Control } \\
\text { subjects }\end{array}$ & 168 & 40 & $\begin{array}{c}208 \\
(52 \times 4)\end{array}$ & 52 \\
\hline $\begin{array}{l}\text { Osteopor } \\
\text { otic } \\
\text { subjects }\end{array}$ & 178 & 46 & $\begin{array}{c}224 \\
(56 \times 4)\end{array}$ & 56 \\
\hline
\end{tabular}

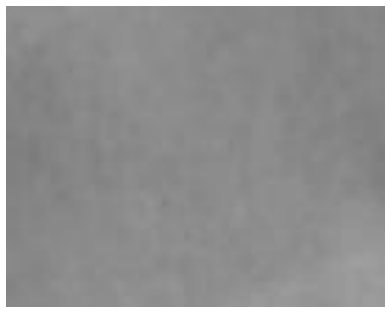

(a)

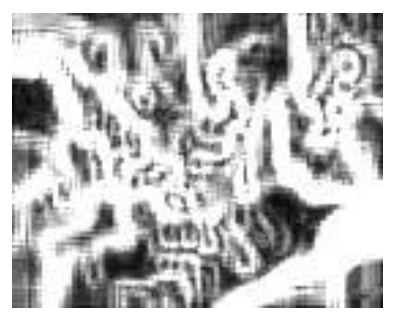

(b)
Figure. 4 Pre-processing results: (a) Abnormal image and (b) Pre-processed image

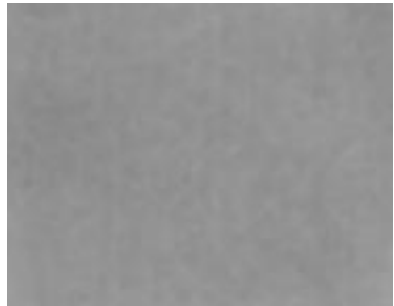

(a)

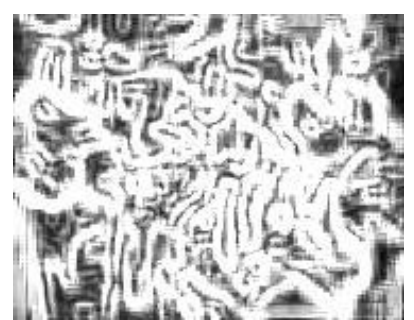

(b)
Figure. 5 Pre-processing results: (a) Normal image and (b) Pre-processed image

the output edges of a two-phase NPG-PCA. There is also a model of sharpening filter along with the range filter to enhance the texture feature at the edges.The results are obtained from a preprocessed abnormal image, as shown in Fig. 4. Noise is present in the input cropped abnormal X-ray image; as a result, the trabecular bone texture feature cannot visualize the bone structure in the image (a). After pre-processing, however, the proposed techniques can reveal the internal trabecular structure in (b). As the input image (a) is an abnormal image, its trabecular bone spacing is greater and its bone density is lower, as depicted in (b). The trabecular bone is more porous and is visible on the pre-processed abnormal X-ray image as dark spots.

In contrast, Fig. 5 shows results for a normal input image as in (a) and illustrates the microarchitecture of the internal trabecular bone as in (b). Since the input image (a) is a normal image, its trabecular bone spacing is smaller and its bone density is greater as shown in (b). Normal X-rays contain more trabecular
Table 2. The PSNR and SSIM of different level of noise in the input images.

\begin{tabular}{|c|c|c|}
\hline $\begin{array}{c}\text { Standard } \\
\text { deviation } \boldsymbol{\sigma}\end{array}$ & $\begin{array}{c}\text { Average } \\
\text { PSNR } \\
\text { (dB) }\end{array}$ & Average SSIM \\
\hline 5 & 38.78 & 0.9853 \\
\hline 10 & 35.74 & 0.9456 \\
\hline 15 & 32.78 & 0.9132 \\
\hline 20 & 30.56 & 0.8978 \\
\hline 25 & 28.95 & 0.8765 \\
\hline 30 & 27.98 & 0.8718 \\
\hline
\end{tabular}

bone density than abnormal X-rays, those affected by osteoporosis. For better enhancement of the trabecular bone structure of X-ray images, the proposed overall pre-processing method works for smaller values of noise variance $\sigma^{2}$ between 1 and 10 .

Table 2. demonstrate the peak signal-to-noise ratio (PSNR) measured in decibels and the structural similarity index (SSIM) of the output of the preprocessed image with the presence of noise at different levels of the input images. As a result, the noise level in input image is estimated as $\sigma$.The PSNR and SSIM values decrease when the input image noise level increases. In spite of this, the proposed preprocessing technique works well within the moderate value of the $\sigma$.

\subsection{Feature extraction and dimension reduction}

The texture feature details in the pre-processed image cannot be clearly observed, however, the proposed technique in this paper is able to extract texture features more effectively, to distinguish normal from abnormal trabecular bone of the lumbar spine, L1-L4. In one level 2D-DWT, different packets of wavelets are applied to the preprocessed image, yielding four different coefficients, like the approximated $\mathrm{A}_{\mathrm{C}}$ is the same as the input image, $\mathrm{H}_{\mathrm{C}}$ is the texture variation along with horizontal directions, $\mathrm{V}_{\mathrm{C}}$ with vertical directions, and $\mathrm{D}_{\mathrm{C}}$ with diagonal variations of structural information, as shown in Fig. 6 (a)-(d) abnormal image and Fig. 7 (a)(d) normal image. In this study, however, only $\mathrm{H}_{\mathrm{C}}$ and $\mathrm{V}_{\mathrm{C}}$ are considered for further processing. The texture information provided by these two DWT coefficients is sufficient to obtain significant features. The structural density of $\mathrm{H}_{\mathrm{C}}$ and $\mathrm{V}_{\mathrm{C}}$ of normal and abnormal images clearly differentiates between them. A selection of strong edges and boundaries and their similarities are utilized as shown in Fig. 8 (a)-(b) abnormal image and Fig. 9 (a)-(b) normal image, which are respectively the 2-D edge Gaussian detection filter response of inputs $H_{C}$ and $V_{C}$. The filter smoothes the intensity variations in $\mathrm{H}_{\mathrm{C}}$ and $\mathrm{V}_{\mathrm{C}}$ 


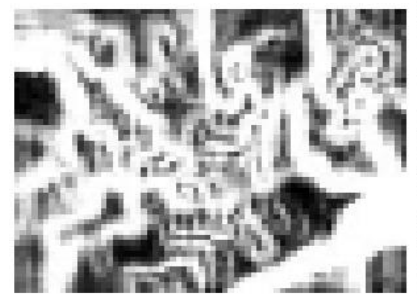

(a)

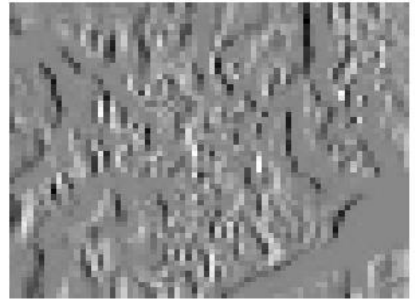

(c)

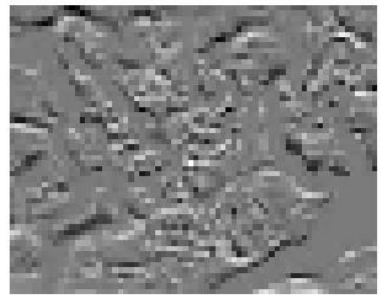

(b)

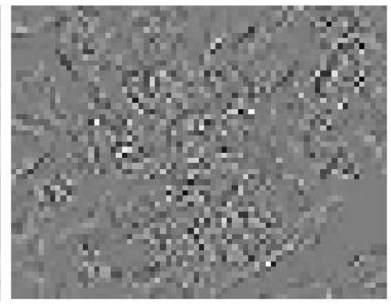

(d)
Figure. 6 DWT output of abnormal image: (a) $\mathrm{A}_{\mathrm{C}}$, (b) $\mathrm{H}_{\mathrm{C}}$, (c) $\mathrm{V}_{\mathrm{C}}$, and (d) $\mathrm{D}_{\mathrm{C}}$

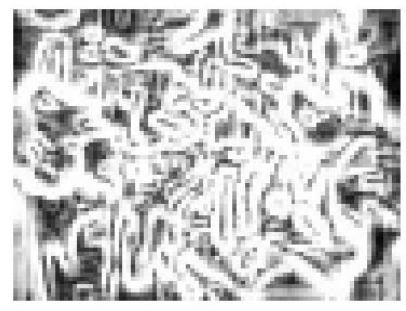

(a)

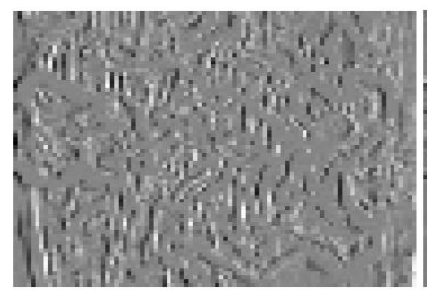

(c)

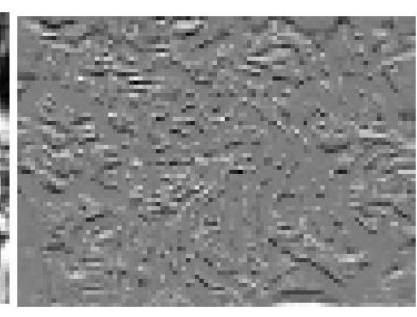

(b)

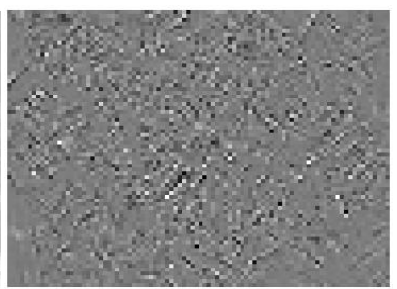

(d)
Figure. 7 DWT output of normal image: (a) $A_{C}$, (b) $\mathrm{H}_{\mathrm{C}}$, (c) $\mathrm{V}_{\mathrm{C}}$, and (d) $\mathrm{D}_{\mathrm{C}}$

coefficients, in addition to highlighting the extreme edges in local areas. Thus, the responses illustrate the edge deviations along the x-axis, which is useful for detecting horizontal edges, and similarly along the $y-$ axis for vertical edges. The filter contains Gaussian and its derivative, so it produces extremum responses that are useful for identifying maximum and minimum texture values in an image.

As a result of applying Eqs. (15) and (16), the magnitudes of the gradient image (a) abnormal and (b) normal are obtained as shown in Fig. 10. With intensity levels, magnitude provides precise edge deviations in the image texture. It estimates every gradient in the image, and its direction reflects how rapidly the image changes. Through the larger gradient rates, the intensity levels become probable

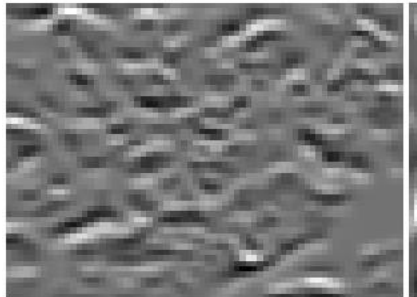

(a)

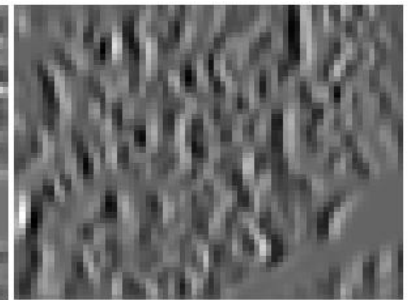

(b)
Figure.8 Filter response on abnormal: (a) $\mathrm{H}_{\mathrm{C}}$ and (b) $\mathrm{V}_{\mathrm{C}}$

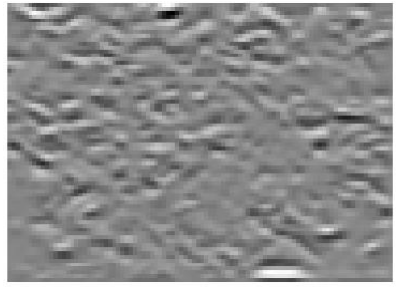

(a)

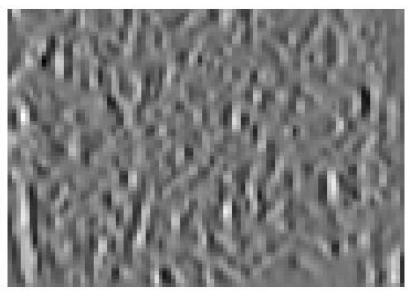

(b)
Figure.9 Filter response on normal: (a) $\mathrm{H}_{\mathrm{C}}$ and (b) $\mathrm{V}_{\mathrm{C}}$

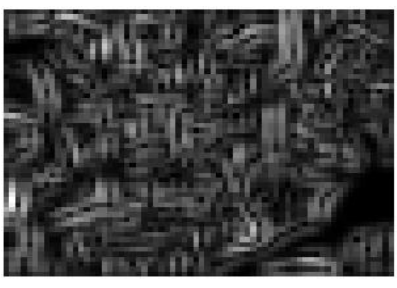

(a)

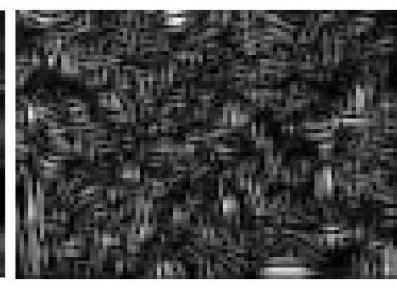

(b)
Figure. 10 Magnitude of gradient image: (a) abnormal and (b) normal

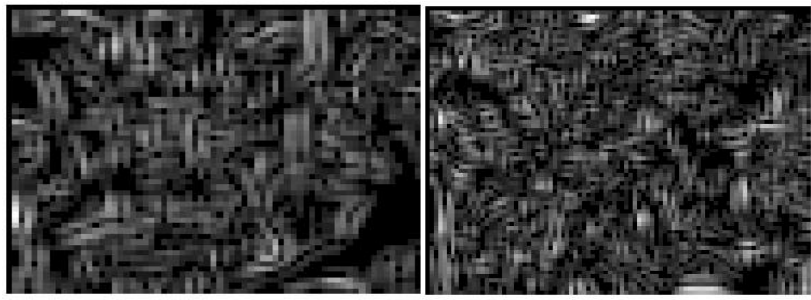

Figure. 11 Texture image after thinning Process: (a) abnormal and (b) normal

edge intensity levels, and the edges are tracked down in the direction perpendicular to the gradient direction. In this way, the proposed feature extraction system is able to derive appropriate structural patterns from abnormal and normal images.

Fig. 11 illustrates the texture image after thinning (a) abnormal (b) normal. The pixel intensity less than the threshold is suppressed, whereas the pixel intensity greater than the threshold is preserved to decrease the texture feature dimension. The reduced texture feature information is deliberately accumulated on either side of the histogram. The fact that no texture features are present in the center of the histogram provides an additional reduction in feature 
Table 3. Histogram feature dimension reduction table

\begin{tabular}{|c|c|c|}
\hline \multicolumn{3}{|c|}{ Original size of each sample=166×128 -----> } \\
$\mathbf{2 1 2 4 8}$ pixels \\
\hline Wave Name & $\begin{array}{c}\text { Computation Time } \\
(\mathrm{sec})\end{array}$ & $\begin{array}{c}\text { Histogram } \\
\text { Feature bins } \\
\text { of Each } \\
\text { Sample }\end{array}$ \\
\hline Haar & 200 & 25 \\
\hline Db1 & 155 & 10 \\
\hline Db2 & 160 & 10 \\
\hline Db3 & 175 & 9 \\
\hline Db4 & 190 & 9 \\
\hline
\end{tabular}

dimension, thereby saving memory for stocking up the trained data vector.

Table 3 illustrates the texture bin histogram of each sample image with different wavelets. However, the input image size is $(166 \times 128) 21248$ pixels ,a technique such as thinning is used to reduce the dimensions of pixels. In contrast to Daubechies wavelets, the Haar wavelet uses a rectangular basis function, which is not differentiable and produces coarse pixel information only at the edges. Thus, a greater number of texture features must be selected for a better classification, requiring more computation time than the Daubechies wavelet. Since Daubechies uses the continuous basis function, which is differentiable, and provides fine pixel information at the edges, there is a need for fewer histogram bins to classify the images into normal and abnormal. As the order of the Daubechies family wavelets increases, the texture information becomes more precise but computation time increases, for better classification, the number of features is less.

\subsection{Classification result}

The Table 4. shows how system performance can be evaluated using a confusion matrix with different classifiers. A detailed analysis of the database, as explained in Table 1, is used for testing the proposed system. DT and RF have less False Positives (FP) and False Negatives (FN) and reduce over fitting in decision making, which increases accuracy, flexibility and works well during classification into normal and abnormal categories. In addition, it introduces missing values in the features database, so no normalization is required. The overall performance of the system leads to better True Positives (TP) and True Negatives (TN) with 5-fold cross-validation as compared to the other classification techniques. A 5-fold cross-validation with DT and RF classifiers yields good accuracy for L1-L4 in Table 5.
Table 4. Evaluation of different classifiers for 5-fold

\begin{tabular}{|c|c|c|c|c|}
\hline Classifiers & TP & FP & TN & FN \\
\hline KNN & 125 & 99 & 99 & 109 \\
\hline DA & 138 & 86 & 85 & 123 \\
\hline NB & 174 & 50 & 152 & 56 \\
\hline DT & 223 & 01 & 206 & 02 \\
\hline SVM & 128 & 96 & 85 & 123 \\
\hline RF & 222 & 02 & 206 & 02 \\
\hline
\end{tabular}

Table 5. Percentage of accuracy from L1-L4 with 5-fold cross validation

\begin{tabular}{|c|c|c|c|c|}
\hline \multirow{2}{*}{ Classifiers } & \multicolumn{4}{|c|}{ Accuracy (\%) } \\
\cline { 2 - 5 } & L1 & L2 & L3 & L4 \\
\hline DT & 99.29 & 99.31 & 99.28 & 99.32 \\
\hline RF & 99.04 & 99.10 & 99.09 & 99.05 \\
\hline
\end{tabular}

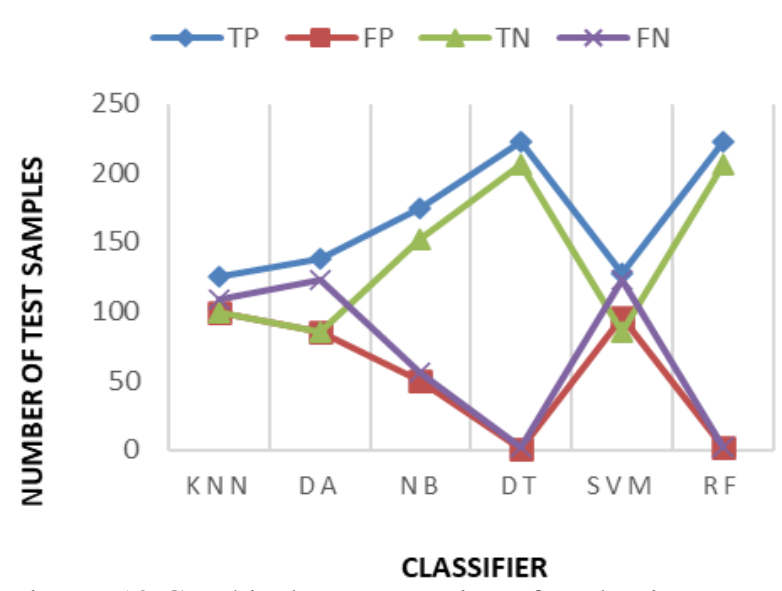

Figure. 12 Graphical representation of evaluation system

The Fig. 12 illustrates the graphical representation of system assessment with different classifiers for 5fold cross validation. As a result, DT and RF achieves the smallest FP and FN and helps the system to be more accurate.

Table 6 illustrates the average performance of the system using different classification techniques for 5fold cross-validation for lumbar spine L1-L4. Sensitivity SN [28] of the system measures the correctness of the test sample, which has the disease (abnormal) and produces few false negatives. Specificity (SP) [28]is the ability to correctly classify the test sample as being free from disease (normal) and producing not many false positives. F1-Score (F1-S) [29] measure the test sample accuracy in terms of FP and FN, which is helpful to measure system accuracy in particular for uneven class distribution. PPV (positive predictive value) is the percentage that actually has the disease if a positive test sample is obtained. NPV (negative predictive values) is the percentage of negative test samples without disease. According to all the above- 
Table 6. Average performance of different classifiers for 5-fold cross validation in (\%)

\begin{tabular}{|c|c|c|c|c|c|c|}
\hline Classifiers & SN & SP & F1-S & PPV & NPV & Accuracy \\
& & & & & & \\
\hline KNN & 53.51 & 50 & 54.57 & 55.80 & 47.59 & 51.85 \\
\hline DA & 52.87 & 49.70 & 56.90 & 61.60 & 40.86 & 51.62 \\
\hline NB & 75.65 & 75.24 & 76.63 & 77.67 & 73.07 & 75.46 \\
\hline DT & $\mathbf{9 9 . 1 1}$ & $\mathbf{9 9 . 5 0}$ & $\mathbf{9 9 . 3 0}$ & $\mathbf{9 9 . 5 0}$ & $\mathbf{9 9 . 0 0}$ & $\mathbf{9 9 . 3 0}$ \\
\hline SVM & 50.99 & 46.96 & 53.89 & 57.14 & 40.86 & 49.30 \\
\hline RF & $\mathbf{9 9 . 1 0}$ & $\mathbf{9 9 . 0 3}$ & $\mathbf{9 9 . 1 0}$ & $\mathbf{9 9 . 1 0}$ & $\mathbf{9 9 . 0 0}$ & $\mathbf{9 9 . 0 7}$ \\
\hline
\end{tabular}

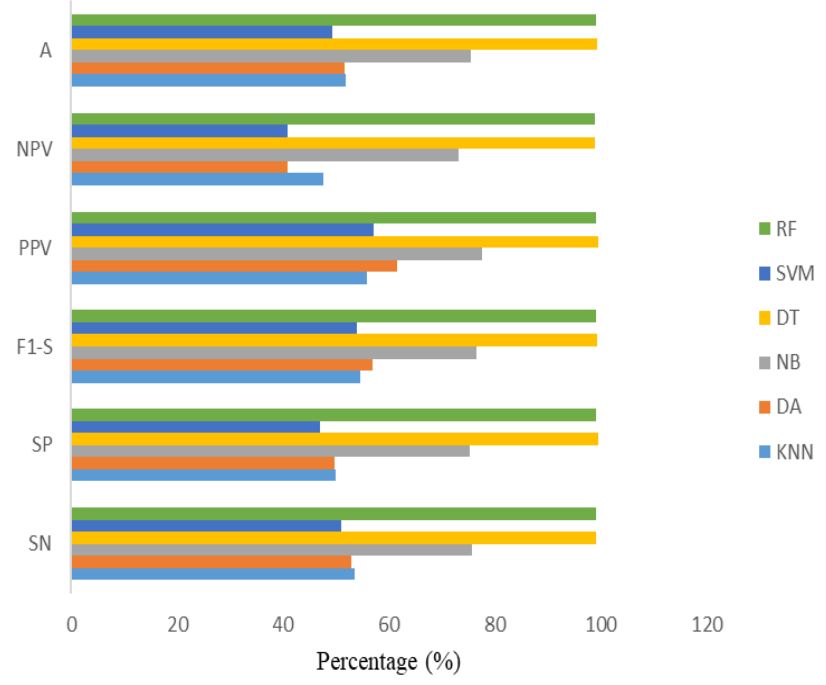

Figure. 13 Average performance graphical representation

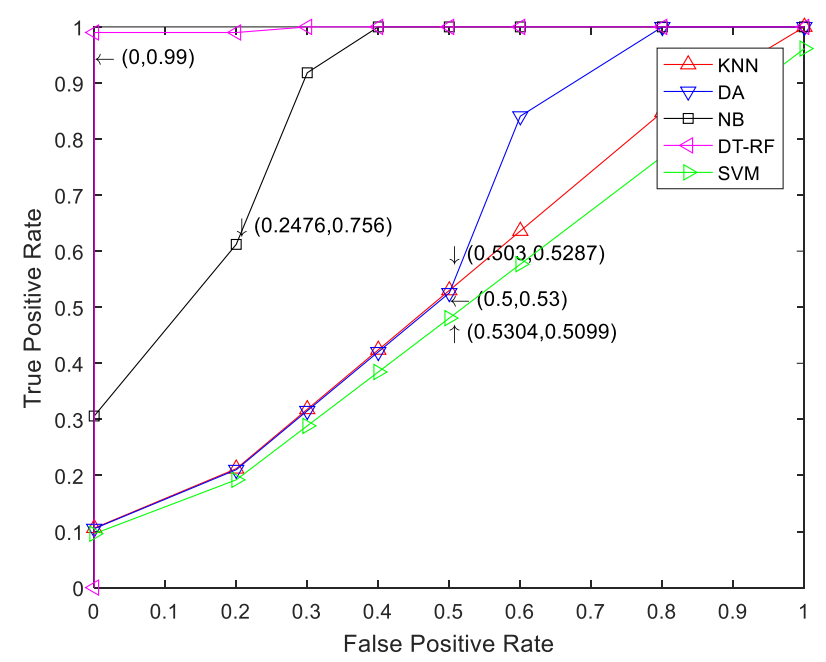

Figure. 14 ROC curve for different classifier

mentioned measures, the DT and RF techniques attain the best classifications and overall, the system measures the trueness of the test samples accurately. Fig. 13 shows the average performance from (L1-L4) of different classifiers based on 5-fold crossvalidation and DT and RF perform better than the other classifiers.

Fig. 14 is the graphical representation of the overall correctness of TP versus FP rate in terms of receiver operating characteristic (ROC) [30] curve for the different classifiers. The DT and RF curves occupy $99 \%$ of the region, resulting in the best classification of abnormal and normal images of test samples. Table 7 compares the performance of different methods and reveal that the proposed method attains the better one compared to the other methods. Because the overall proposed system has pre-processing and significant texture feature extraction methods that will allow it to detect osteoporosis using more accurate classification methods. As a result, the proposed system has a better accuracy, sensitivity, specificity, and F1-score than the system proposed in [20].

\section{Conclusion and future work}

This investigation demonstrated that two-stage NPG-PCA successfully preserves the fine structures in the image while smoothing the noise. An arrangement of pixels and their neighbors has been represented in a vector variable in the system model, and the de-noising of the pixel has been adapted into the evaluation of the variable based on noisy observations. Especially in X-ray images, it offers a competitive de-noising way out compared with other de-noising algorithms. The enhancement technique that is used in this work makes it possible to visualize the fine internal trabecular bone structure. The preprocessing helps experimentally to extract the features of trabecular bone structure effectively. 2DDWT combined with 2D edge detection provided very fine texture information and brought in the extreme edge detection to maintain the considerable feature in order to reduce its dimension. Among the many advantages of this development, the procedure is that it dramatically reduces database size for texture features. Our study evaluated various machine learning classification methods on spine $\mathrm{X}$ ray images of lumbar L1-L4 in order to build up a prediction model to classify the normal and abnormal of osteoporosis. Comparatively, the DT and RF provide good classification results and provide an average accuracy of $99.18 \%$. Particularly, this work helps patients at high risk of osteoporosis, enabling 
Table 7. Performance comparison with different methods

\begin{tabular}{|c|c|c|c|c|c|c|}
\hline $\begin{array}{c}\text { Methods and } \\
\text { features }\end{array}$ & $\begin{array}{c}\text { Imaging } \\
\text { modalities }\end{array}$ & Classifiers & $\begin{array}{c}\text { Accuracy } \\
(\%)\end{array}$ & $\begin{array}{c}\text { Sensitivity } \\
(\%)\end{array}$ & $\begin{array}{c}\text { Specificity } \\
(\%)\end{array}$ & $\begin{array}{c}\text { Cross } \\
\text { validation }\end{array}$ \\
\hline $\begin{array}{c}\text { Fractal Analysis } \\
{[6]}\end{array}$ & $\mathrm{CT}$ & k-NN & 81 & 78 & 90 & 3-fold \\
\hline $\begin{array}{c}\text { Morphometric } \\
{[10]}\end{array}$ & CT & k-NN & 88.3 & 92.5 & 83.3 & 10 -fold \\
\hline Multifractal [11] & $\begin{array}{c}\text { CT and } \\
\text { MRI }\end{array}$ & SVM & 95.2 & $\mathrm{~N}$ & $\mathrm{~N}$ & 10-fold \\
\hline $\begin{array}{l}\text { Morphometric } \\
\text { with global } \\
\text { descriptors [12] }\end{array}$ & CT & SVM & 82.0 & $\mathrm{~N}$ & $\mathrm{~N}$ & 10-fold \\
\hline $\begin{array}{l}\text { Morphometric } \\
\text { with Fourier } \\
\text { descriptors [13] }\end{array}$ & MRI & k-NN & 90.58 & $\mathrm{~N}$ & $\mathrm{~N}$ & 10-fold \\
\hline $\begin{array}{c}\text { MBO-ANN } \\
\text { approach [14] }\end{array}$ & $\begin{array}{l}\text { Person } \\
\text { attribute } \\
\text { values }\end{array}$ & Hybrid & $97.9 \pm 0.14$ & $98.33 \pm 0.03$ & $98.33 \pm 0.03$ & 10-fold \\
\hline $\begin{array}{l}\text { 3D feature with } \\
\text { vBMD [17] }\end{array}$ & CT & Random forest & - & 77 & 78 & 4-fold \\
\hline $\begin{array}{l}\text { Regression based } \\
\text { model [18] }\end{array}$ & $\begin{array}{l}\text { DEXA and } \\
\text { CT }\end{array}$ & Random forest & 82 & 84.4 & 72.7 & 2.7 -fold \\
\hline CNN [19] & $\mathrm{CT}$ & $\begin{array}{c}\text { BCC-net } \\
\text { MS-net }\end{array}$ & 76.5 & $\mathrm{~N}$ & $\mathrm{~N}$ & $\begin{array}{l}\text { 7-fold } \\
2 \text {-fold }\end{array}$ \\
\hline DCN [20] & $\begin{array}{c}\text { DEXA and } \\
\text { X-ray }\end{array}$ & $\begin{array}{c}\text { SVMC, KNN, } \\
\text { RFC }\end{array}$ & 71 & 81 & 60 & 5 -fold \\
\hline $\begin{array}{c}\text { Statistical } \\
\text { Quantitative } \\
\text { Analysis [21] } \\
\end{array}$ & $\begin{array}{l}\text { MRI and } \\
\text { DEXA }\end{array}$ & $\mathrm{N}$ & $\mathrm{N}$ & 94 & 60 & 2 -fold \\
\hline Proposed & $\begin{array}{l}\text { X-ray and } \\
\text { DEXA } \\
\text { Report } \\
\end{array}$ & $\begin{array}{l}\text { DT } \\
\text { RF }\end{array}$ & $\begin{array}{l}99.30 \\
99.07\end{array}$ & $\begin{array}{l}99.11 \\
99.10\end{array}$ & $\begin{array}{l}99.50 \\
99.03\end{array}$ & $\begin{array}{l}\text { 5-fold } \\
\text { 5-fold }\end{array}$ \\
\hline
\end{tabular}

them to detect it earlier and help the persons who require medical suggestions, prevent the disease, and achieve a better medical report. It is anticipated that future assessments will progress the performance of the training with another outfit technique in larger populations. This will be more informative for quantitative analysis of the trabecular bone structure of X-ray images of lumbar spine to avoid osteoporosis and to be more cost-effective.

\section{Conflicts of interest}

The authors declare no conflict of interest.

\section{Author contributions}

The paper conceptualization, methodology, software, resources, writing-original draft preparation ,writing - review and editing, visualization, investigation have done by $1^{\text {st }}$ author . The Supervision and project administration have done by $2^{\text {nd }}$ author. The data curation, validation and formal analysis have done by $3^{\text {rd }}$ author. The X-ray (raw-data) and DEXA report provided by $3^{\text {rd }}$ author.

\section{Acknowledgement}

"This work was supported by Dr. A Ramalingaiah M.S (ortho)Orthopedic Doctor, Bangalore. No.271 Abhilasha Orthopaedic Hospital 5th Block 100ft Rd Banashankari 3rd Stage Bangalore - 560085, Bangalore".

\section{References}

[1] J. A. Peterson, "Osteoporosis overview", Geriatric Nursing, Vol. 22, No.1, pp. 1723,2001 .

[2] R. C. Gonzalez, "Richard E. woods", Digital image processing, Vol. 2, pp. 550-570, 2002.

[3] A. Pizurica, W. Philips, I. Lemahieu, and M. Acheroy, "A joint inter-and intrascale statistical model for Bayesian wavelet-based image denoising", IEEE Transactions on Image Processing, Vol. 11, No. 5, pp. 545-557,2002.

[4] J. L. Starck, E. J. Candès, and D. L. Donoho, "The curvelet transform for image denoising", IEEE Transactions on image processing, Vol. 11, No. 6, pp. 670-684,2002. 
[5] G. Y. Chen and B. Kégl, "Image denoising with complex ridgelets", Pattern Recognition, Vol. 40, No. 2, pp. 578-585, 2007.

[6] Z. Omiotek, R. Dzierżak, and S. Uhlig, "Fractal analysis of the computed tomography images of vertebrae on the thoraco-lumbar region in diagnosing osteoporotic bone damage", In: Proc. of the Institution of Mechanical Engineers, Part $H$ : Journal of Engineering in Medicine, Vol. 233, No. 12, pp. 1269-1281, 2019.

[7] K. H. Nam, I. Seo, D. H. Kim, J. I. Lee, B. K. Choi, and I. H. Han, "Machine Learning Model to Predict Osteoporotic Spine with Hounsfield Units on Lumbar Computed Tomography", Journal of Korean Neurosurgical Society, Vol. 62, No. 4, pp. 442-449, 2019.

[8] M. L. Picazo, A. M. Baro, L. M. D. R. Barquero, S. D. Gregorio, Y. Martelli, J. Romera, M. Steghöfer, M. A. G. Ballester, and L. Humbert, "3-D Subject-Specific Shape and Density Estimation of the Lumbar Spine from a Single Anteroposterior DXA Image Including Assessment of Cortical and Trabecular Bone", IEEE Transactions on Medical Imaging, Vol. 37, No. 12, pp. 2651-2662, 2018.

[9] A. S. Shaker, "Detection and Segmentation of Osteoporosis in Human Body using Recurrent Neural Network", International Journal of Advanced Science and Technology, Vol. 29, No. 2, pp. 1055-1066, 2020.

[10] H. Yousefi, E. Salehi, O. S. Sheyjani, and H. Ghanaatti, "Lumbar Spine Vertebral Compression Fracture Case Diagnosis Using Machine Learning Methods on CT images", In: Proc. of 4th International Conference on Pattern Recognition and Image Analysis (IPRIA) IEEE, Tehran, Iran, pp. 179-184, 2019.

[11] R. Korchiyne, R. Touahni, S. M. Farssi, A. Sbihi, M. Issam, M. T. Alaoui, and E. I. Y. Merabet, "New Approach Based on Multifractal Spectrum Features for Detection of Osteoporosis", In: Proc. of International Symposium on Advanced Electrical and Communication Technologies (ISAECT), Rabat, Morocco, Vol. 2018, pp. 1-5,2018.

[12] Y. Wang, J. Yao, J. E. Burns, and R. Summers, "Osteoporotic and neoplastic compression fracture classification on longitudinal CT", In: Proc. of IEEE 13th International Symposium on Biomedical Imaging (ISBI), Prague, pp. 11811184, 2016.

[13] L. F. Pereira, R. M. Reis, G. A. Metzner, R. M. Rangayyan, M. H. N. Barbosa, and P. M. A. Marques, "Classification of vertebral compression fractures in magnetic resonance images using shape analysis", In: Proc. of IEEE E-Health and Bioengineering Conference (EHB), Iasi, pp. 1-4, 2015.

[14] D. Devikanniga and R. J. S. Raj, "Classification of osteoporosis by artificial neural network based on monarch butterfly optimisation algorithm", Healthcare technology letters, Vol. 5, No.2, pp. 70-75,2018.

[15] B. Zhang, K. Yu, Z. Ning, K. Wang, Y. Dong, X. Liu, S. Liu, J. Wang, C. Zhu, Q. Yu, Y. Duan, S. Lv, X. Zhang, Y. Chen, X. Wang, J. Shen, and J. Peng, "Deep learning of lumbar spine X-ray for osteopenia and osteoporosis screening: A multicenter retrospective cohort study", Bone, Vol. 140, p. 115561, 2020.

[16] K. Murata, K. Endo, T. Aihara, H. Suzuki, Y. Sawaji, Y. Matsuoka, H. Nishimura, T. Takamatsu, T. Konishi, A. Maekawa, and H. Yamauchi, "Artificial intelligence for the detection of vertebral fractures on plain spinal radiography", Scientific Reports, Vol. 10, No. 1, pp. 1-8, 2020.

[17] A. Valentinitsch, S. Trebeschi, J. Kaesmacher, C. Lorenz, M. T. Löffler, C. Zimmer, T. Baum, and J. S. Kirschke, "Opportunistic osteoporosis screening in multi-detector CT images via local classification of textures", Osteoporosis international, Vol. 30, No. 6, pp. 1275-1285, 2019.

[18] J. M. Czum, "Simulated Lumbar Dual-Energy X-Ray Absorptiometry with Machine Learning Algorithms: Why Radiologists Who Interpret Abdominal CTs Should Care", Journal of the American College of Radiology, Vol. 16, No. 10, pp. 1471-1472, 2019.

[19] C. Tang, W. Zhang, H. Li, L. Li, Z. Li, A. Cai, L. Wang, D. Shi, and B. Yan, "CNN-based automatic detection of bone conditions via diagnostic CT images for osteoporosis screening", arXiv preprint arXiv:1910.06777, 2019.

[20] S. Lee, E. K. Choe, H. Y. Kang, J. W. Yoon, and H. S. Kim, "The exploration of feature extraction and machine learning for predicting bone density from simple spine X-ray images in a Korean population", Skeletal Radiology, Vol. 49, No. 4, pp. 613-618, 2020.

[21] M. M. Saad, A. T. Ahmed, K. E. Mohamed, and M. R. Habba, "Role of lumbar spine signal intensity measurement by MRI in the diagnosis of osteoporosis in post-menopausal women", Egyptian Journal of Radiology and Nuclear Medicine, Vol. 50, No. 1, pp. 1-7, 2019.

[22] S. Mallat and S. Zhong, "Characterization of signals from multiscale edges", IEEE 
Transactions on Pattern Analysis and Machine Intelligence, Vol. 14, No. 7, pp. 710-732, 1992.

[23] N. Otsu, "A threshold selection method from gray-level histograms", IEEE Transactions on Systems, Man, and Cybernetics, Vol. 9, No. 1, pp. 62-66, 1979.

[24] U. Ferizi, S. Honig, and G. Chang, “Artificial intelligence, osteoporosis and fragility fractures", Current Opinion in Rheumatology, Vol. 31, No. 4, p. 368, 2019.

[25] A. F. M. Alkarkhi, and W. A. A. Alqaraghuli, Easy statistics for food science with R, Vol.1, Academic Press, Elsevier ,2018.

[26] M. S. Mushtaq and A. Mellouk, Quality of Experience Paradigm in Multimedia Services: Application to OTT Video Streaming and VoIP Services, Vol. 1, Elsevier, 2017.

[27] D. D. Muresan and T. W. Parks, "Adaptive principal components and image denoising", In: Proc. of 2003 International Conference on Image Processing (Cat. No. O3CH37429) IEEE, Vol. 1, pp. 1-101, 2003.

[28] R. Parikh, A. Mathai, S. Parikh, G. C. Sekhar, and R. Thomas, "Understanding and using sensitivity, specificity and predictive values", Indian journal of ophthalmology, Vol. 56, No. 1, p. 45, 2008.

[29] https://blog.exsilio.com/all/accuracy-precisionrecall-f1-score-interpretation-of-performancemeasures/

[30] A. M. Baese and V. J. Schmid, Pattern recognition and signal analysis in medical imaging, Vol. 1, Academic Press, Elsevier, 2014.

[31] I. Bankman, Handbook of medical image processing and analysis, Vol. 1, Academic Press, Elsevier, 2008. 